\title{
COVERINGS OF LOCALLY CONFORMALLY KÄHLER COMPLEX SPACES
}

\author{
OVIDIU PREDA AND MIRON STANCIU
}

\begin{abstract}
In this paper, we study the properties of coverings of locally conformally Kähler (LCK) spaces with singularities. We begin by proving that a space is LCK if any only if its universal cover is Kähler, thereby generalizing a result from [IP]. We then show that a complex space which projects over an LCK space with discrete fibers must also carry an LCK structure.
\end{abstract}

\section{CONTEnts}

1. Introduction 1

2. Integrating closed 1-forms on complex spaces 3

3. Locally conformally Kähler spaces 6

4. Mappings with discrete fibers between LCK spaces 9

References 14

\section{INTRODUCTION}

The notion of locally conformally Kähler (LCK) manifolds was first introduced by I. Vaisman [Vai]. As the name implies, they are manifolds endowed with a complex structure and a non-degenerate two-form which is locally conformal to a Kähler form. They can equivalently be defined as quotients of Kähler manifolds by a discrete group of homotheties of the Kähler form. Since their introduction, they have been extensively studied (see $[\mathrm{OV}]$ for an overview of the field and e.g. [I], [MMOr], [Ot] for some recent results).

Our main goal is to study which fundamental properties of LCK structures still hold true in the singular setting. The first to introduce the definition of Kähler form on complex spaces with singularities was Grauert in [G]

Date: January 22, 2020.

Ovidiu Preda was partially supported by a grant of Ministry of Research and Innovation, CNCS - UEFISCDI, project number PN-III-P1-1.1-PD-2016-0182, within PNCDI III.

Keywords: Complex spaces, locally conformally Kähler.

2010 Mathematics Subject Classification: 32C15, 53C55. 
and this study was continued by Moishezon in [Mo]. Notable results about the stability of Kähler structures were obtained by [Var]. Recently, in [IP], the authors gave a natural definition of the same type for LCK spaces and studied the properties of its universal cover, proving, using resolutions of singularities, that, if the space has quotient singularities, the result from the smooth case holds true i.e. the universal cover is Kähler. They conjectured that this should hold without the additional condition.

One of the key difficulties in working with non-smooth complex spaces is that there is no workable definition for $(p, q)$-forms (for a study of differential forms on singular spaces, see [P, pp. 375-388]).

In this paper, we firstly improve the main result from [IP] by removing any requirement on the singularity types and in fact finding a way to not mention the singularities at all. Instead, we develop a method for defining closed 1-forms on complex spaces (without first saying what a 1-form is) and integrating them along curves. We are then able to essentially use the same proof as in the smooth case for recovering the result we wanted.

Finally, we show that the characterization we found is useful i.e. by passing to the universal cover, we can prove new results about coverings of LCK spaces with discrete fibers.

In Section 2, we start by giving the definition of closed 1-forms on complex spaces and proving that a few basic results about their integration along curves that are true in the smooth case also hold in the singular case.

In Section 3, we are then able to improve the result from [IP] by completely recovering the characterization of LCK spaces as quotients of Kähler spaces by homotheties of its Kähler metric. More precisely, we prove:

Theorem 3.10. Let $X$ be a complex space. Then $X$ admits an LCK metric if and only if its universal covering $\tilde{X}$ admits a Kähler metric such that the deck automorphisms act on $\tilde{X}$ by positive homothethies of the Kähler metric.

In Section 4, using the above characterization, we generalize to the locally conformal setting a result by [Vaj] about maps with discrete fibers into Kähler spaces. Specifically, we prove:

Theorem 4.1. Let $g: X \longrightarrow Y$ be a holomorphic map between complex spaces with discrete fibers and assume $(Y, \omega, \theta)$ is LCK. Then $X$ also carries an LCK structure. 


\section{Integrating ClOSED 1-FORMS ON COMPLEX SPACES}

Let $X$ be a connected complex space of dimension $n$.

\section{Definition 2.1:}

1) Denote by

$$
\begin{array}{r}
\tilde{Z}^{1}(X)=\left\{\left(U_{\alpha}, f_{\alpha}\right)_{\alpha \in A} \mid\left(U_{\alpha}\right)_{\alpha} \text { a covering of } X, f_{\alpha}: U_{\alpha} \longrightarrow \mathbb{C}\right. \text { smooth } \\
\text { and } \left.f_{\alpha}-f_{\beta} \text { locally constant on } U_{\alpha} \cap U_{\beta}, \forall \alpha, \beta \in A\right\} .
\end{array}
$$

We define an equivalence relation on $\tilde{Z}^{1}(X)$ by

$$
\left(U_{\alpha}, f_{\alpha}\right)_{\alpha \in A} \sim\left(V_{\beta}, h_{\beta}\right)_{\beta \in B} \Longleftrightarrow\left(U_{\alpha}, f_{\alpha}\right)_{\alpha \in A} \cup\left(V_{\beta}, h_{\beta}\right)_{\beta \in B} \in \tilde{Z}^{1}(X) .
$$

2) We define the space of smooth closed 1-forms on $X$ to be the quotient space $Z^{1}(X)=\tilde{Z}^{1}(X) / \sim$. An element $\theta \in Z^{1}(X)$ is called a smooth closed 1-form.

3) An element $\theta \in Z^{1}(M)$ is called exact if $\theta=\widehat{(X, f)}$ for a smooth $f$ : $X \longrightarrow \mathbb{C}$. In this case, we make the notation $\theta=d f$.

Remark 2.2: $\quad$ Outside the singular locus $\operatorname{Sing}(X)$, the above definition coincides with the usual one on smooth manifolds.

Similar to the smooth case, one can define the notion of pullback for closed 1-forms:

Definition 2.3: Let $\phi: X \longrightarrow Y$ be a smooth map between complex spaces and $\theta \in Z^{1}(Y)$. Let $\theta=\overline{\left(U_{\alpha}, f_{\alpha}\right)_{\alpha \in A}}$.

We denote by $\phi^{*} \theta=\overline{\left(\phi^{-1}\left(U_{\alpha}\right), \phi^{*} f_{\alpha}\right)_{\alpha \in A}} \in Z^{1}(X)$, and call it the pullback of $\theta$ via $\phi$. Indeed, one can check that this is well defined and that it coincides with the usual definition on smooth manifolds.

Proposition 2.4: Let $\phi: X \longrightarrow Y$ be a smooth map between complex spaces and $f: Y \longrightarrow \mathbb{C}$ smooth.

Then

$$
\phi^{*} d f=d \phi^{*} f .
$$

Proof. The statement follows directly from the definitions.

Proposition 2.5: Let $\gamma:[0,1] \longrightarrow X$ be a curve on $X$ and take $\theta \in Z^{1}(X)$, $\theta=\overline{\left(U_{\alpha}, f_{\alpha}\right)_{\alpha \in A}}$.

Choose a partition $0=a_{0}<a_{1}<\ldots<a_{m}=1$ such that $\gamma\left(\left[a_{k}, a_{k+1}\right]\right) \subset$ $U_{\alpha_{k}}$ for some $\alpha_{k} \in A$.

Then the number

$$
\int_{\gamma,\left(U_{\alpha}, f_{\alpha}\right)_{\alpha},\left(a_{0}, \ldots, a_{m}\right)} \theta=\sum_{k=0}^{m}\left(f_{\alpha_{k}}\left(\gamma\left(a_{k+1}\right)\right)-f_{\alpha_{k}}\left(\gamma\left(a_{k}\right)\right)\right)
$$

only depends on $\gamma$ and $\theta$. 
Proof. Note that, for any fixed $k$, the choice of $\alpha_{k}$ such that $\gamma\left(\left[a_{k}, a_{k+1}\right]\right) \subset$ $U_{\alpha_{k}}$ does not change the result: if $\gamma\left(\left[a_{k}, a_{k+1}\right]\right) \subset U_{\alpha} \cap U_{\beta}$, then $f_{\alpha}-f_{\beta}$ is constant on $\gamma\left(\left[a_{k}, a_{k+1}\right]\right)$, so

$$
f_{\alpha}\left(\gamma\left(a_{k+1}\right)\right)-f_{\alpha}\left(\gamma\left(a_{k}\right)\right)=f_{\beta}\left(\gamma\left(a_{k+1}\right)\right)-f_{\beta}\left(\gamma\left(a_{k}\right)\right) .
$$

We now prove the independence on the choice of partition of the unit interval. Take two such partitions

$$
\begin{aligned}
& 0=a_{0}<a_{1}<\ldots<a_{m}=1 \\
& 0=b_{0}<b_{1}<\ldots<b_{l}=1
\end{aligned}
$$

and consider the joined partition

$$
0=c_{0}<c_{1}<\ldots<c_{s}=1 .
$$

Fix $0 \leq k<m$. There exist $0 \leq r<p \leq s$ such that $c_{r}=a_{k}$ and $c_{p}=a_{k+1}$. Note that $\gamma\left(\left[c_{t}, c_{t+1}\right]\right) \subset U_{\alpha_{k}}$ for all $r \leq t<p$. Then

$$
\sum_{t=r}^{p-1}\left(f_{\alpha_{k}}\left(\gamma\left(c_{t+1}\right)\right)-f_{\alpha_{k}}\left(\gamma\left(c_{t}\right)\right)\right)=f_{\alpha_{k}}\left(\gamma\left(a_{k+1}\right)\right)-f_{\alpha_{k}}\left(\gamma\left(a_{k}\right)\right)
$$

so, according to the remark at the beginning of the proof, and summing by $k$, we have

Similarly,

$$
\int_{\gamma,\left(U_{\alpha}, f_{\alpha}\right)_{\alpha},\left(a_{0}, \ldots, a_{m}\right)} \theta=\int_{\gamma,\left(U_{\alpha}, f_{\alpha}\right)_{\alpha},\left(c_{0}, \ldots, c_{s}\right)} \theta
$$

$$
\int_{\gamma,\left(U_{\alpha}, f_{\alpha}\right)_{\alpha},\left(b_{0}, \ldots, b_{l}\right)} \theta=\int_{\gamma,\left(U_{\alpha}, f_{\alpha}\right)_{\alpha},\left(c_{0}, \ldots, c_{s}\right)} \theta .
$$

Lastly, we prove the independence on the choice of representative for $\theta$. Take $\theta=\overline{\left(U_{\alpha}, f_{\alpha}\right)_{\alpha \in A}}=\overline{\left(V_{\beta}, h_{\beta}\right)_{\beta \in B}}$. Choose a partition $0=a_{0}<\ldots<$ $a_{k}=1$ of the unit interval such that $\gamma\left(\left[a_{k}, a_{k+1}\right]\right) \subset U_{\alpha_{k}} \cap V_{\beta_{k}}$ for some $\alpha_{k} \in A, \beta_{k} \in B$. Then

$$
f_{\alpha_{k}}\left(\gamma\left(a_{k+1}\right)\right)-f_{\alpha_{k}}\left(\gamma\left(a_{k}\right)\right)=h_{\beta_{k}}\left(\gamma\left(a_{k+1}\right)\right)-h_{\beta_{k}}\left(\gamma\left(a_{k}\right)\right),
$$

so

$$
\int_{\gamma,\left(U_{\alpha}, f_{\alpha}\right)_{\alpha},\left(a_{0}, \ldots, a_{m}\right)} \theta=\int_{\gamma,\left(V_{\beta}, h_{\beta}\right)_{\beta},\left(a_{0}, \ldots, a_{m}\right)} \theta .
$$

Definition 2.6: For any curve $\gamma$ and any 1-form $\theta$, we call the number defined via Proposition 2.5 the integral of $\theta$ along $\gamma$ and denote it by

$$
\int_{\gamma} \theta
$$

Remark 2.7: If the image of $\gamma$ does not intersect the singular locus, the above definition coincides with the regular integral of a 1-form along a curve on a smooth manifold. 
Proposition 2.5 has the following immediate

Corollary 2.8: If $\gamma_{0}, \gamma_{1}$ are two curves with the same endpoints in $X$, $\theta \in Z^{1}(X)$ and there exist $\theta=\overline{\left(U_{\alpha}, f_{\alpha}\right)_{\alpha \in A}}$ and a partition $0=a_{0}<a_{1}<$ $\ldots<a_{m}=1$ with $\gamma_{0}\left(\left[a_{k}, a_{k+1}\right]\right) \cup \gamma_{1}\left(\left[a_{k}, a_{k+1}\right]\right) \subset U_{\alpha_{k}}$ for some $\alpha_{k} \in A$ such that $\gamma_{0}\left(a_{k}\right)=\gamma_{1}\left(a_{k}\right)$ for all $0 \leq k \leq m$, then

$$
\int_{\gamma_{0}} \theta=\int_{\gamma_{1}} \theta
$$

Proposition 2.9: The integral of $\theta$ along $\gamma$ only depends on the homotopy class (with fixed endpoints) of $\gamma$.

Proof. Consider a homotopy $\gamma$ : : $[0,1] \times[0,1] \longrightarrow X, \gamma_{t}(0)=a, \gamma_{t}(1)=b$. We show that

$$
\left([0,1] \ni t \longrightarrow \int_{\gamma_{t}} \theta\right) \text { is locally constant. }
$$

Fix $t_{0} \in[0,1]$. Take $\theta=\overline{\left(U_{\alpha}, f_{\alpha}\right)_{\alpha \in A}}$ with $U_{\alpha}$ coordinate charts and choose a partition $0=a_{0}<\ldots<a_{m}=1$ and an $\epsilon>0$ such that, for all $0 \leq k \leq m-1$,

$$
\gamma\left(\left[a_{k}, a_{k+1}\right] \times\left(t_{0}-\epsilon, t_{0}+\epsilon\right)\right) \subset U_{\alpha_{k}} \text { for some } \alpha_{k} \in A .
$$

By Proposition 2.5, we have:

$$
\begin{aligned}
& \int_{\gamma_{t}} \theta-\int_{\gamma_{t_{0}}} \theta= \\
& \sum_{k=0}^{m-1}\left(f_{\alpha_{k}}\left(\gamma_{t}\left(a_{k+1}\right)\right)-f_{\alpha_{k}}\left(\gamma_{t}\left(a_{k}\right)\right)\right)-\sum_{k=0}^{m-1}\left(f_{\alpha_{k}}\left(\gamma_{t_{0}}\left(a_{k+1}\right)\right)-f_{\alpha_{k}}\left(\gamma_{t_{0}}\left(a_{k}\right)\right)\right)= \\
& \sum_{k=0}^{m-1}\left(f_{\alpha_{k}}\left(\gamma_{t}\left(a_{k+1}\right)\right)-f_{\alpha_{k}}\left(\gamma_{t_{0}}\left(a_{k+1}\right)\right)\right)-\sum_{k=0}^{m-1}\left(f_{\alpha_{k}}\left(\gamma_{t}\left(a_{k}\right)\right)-f_{\alpha_{k}}\left(\gamma_{t_{0}}\left(a_{k}\right)\right)\right)= \\
& \sum_{k=0}^{m-1}\left(f_{\alpha_{k+1}}\left(\gamma_{t}\left(a_{k+1}\right)\right)-f_{\alpha_{k+1}}\left(\gamma_{t_{0}}\left(a_{k+1}\right)\right)\right)-\sum_{k=0}^{m-1}\left(f_{\alpha_{k}}\left(\gamma_{t}\left(a_{k}\right)\right)-f_{\alpha_{k}}\left(\gamma_{t_{0}}\left(a_{k}\right)\right)\right)= \\
& =0, \text { for any } t \in\left(t_{0}-\epsilon, t_{0}+\epsilon\right) .
\end{aligned}
$$

For the third equality, replacing $f_{\alpha_{k}}$ with $f_{\alpha_{k+1}}$ in the first sum is possible, since the curve $s \mapsto \gamma_{s}\left(a_{k+1}\right)$, with $s$ between $t$ and $t_{0}$, is contained entirely in $U_{\alpha_{k}} \cap U_{\alpha_{k+1}}$.

Corollary 2.10: If $X$ is simply connected, every closed 1-form $\theta$ is exact.

Proof. Take $\theta=\overline{\left(U_{\alpha}, f_{\alpha}\right)_{\alpha \in A}}$. Fix $x_{0} \in X$. We define $f: X \longrightarrow \mathbb{C}$,

$$
f(x)=\int_{x_{0}}^{x} \theta:=\int_{\gamma} \theta, \text { where } \gamma \text { is a curve from } x_{0} \text { to } x .
$$

Since $X$ is simply connected and by Proposition 2.9, this does not depend on the choice of $\gamma$. 
We now show that $\theta=d f$ i.e. $f-f_{\alpha}$ is locally constant on $U_{\alpha}$ for all $\alpha \in A$. Choose $x_{\alpha} \in U_{\alpha}$. Then

$$
f(x)=\int_{x_{0}}^{x_{\alpha}} \theta+\int_{x_{\alpha}}^{x} \theta=\int_{x_{0}}^{x_{\alpha}} \theta+f_{\alpha}(x)-f_{\alpha}\left(x_{\alpha}\right),
$$

so

$$
f(x)-f_{\alpha}(x)=\int_{x_{0}}^{x_{\alpha}} \theta-f_{\alpha}\left(x_{\alpha}\right), \forall x \in U_{\alpha},
$$

and the right-hand side is a constant which does not depend on the choice of $x_{\alpha} \in U_{\alpha}$.

\section{LOCALly CONFORMALLY KÄHLER SPACES}

In this section, we give the definitions of the main objects we will be working with, as well as prove a result characterizing LCK spaces via their universal covering, thereby generalizing a well-known property which is true in the smooth case.

The following definition is very slightly adapted from [Mo] to resemble Definition 2.1:

\section{Definition 3.1:}

1) Denote by

$$
\begin{aligned}
\tilde{\mathcal{K}}(X)=\{ & \left(U_{\alpha}, \varphi_{\alpha}\right)_{\alpha \in A} \mid\left(U_{\alpha}\right)_{\alpha} \text { a covering of } X, \varphi_{\alpha}: U_{\alpha} \longrightarrow \mathbb{R} \\
& \text { strongly plurisubharmonic and } \varphi_{\alpha}-\varphi_{\beta}=\operatorname{Re} g_{\alpha \beta} \text { on } U_{\alpha} \cap U_{\beta}, \\
& \text { for some holomorphic } \left.g_{\alpha \beta}: U_{\alpha} \cap U_{\beta} \longrightarrow \mathbb{C}, \forall \alpha, \beta \in A\right\} .
\end{aligned}
$$

We define an equivalence relation on $\tilde{\mathcal{K}}(X)$ by

$$
\left(U_{\alpha}, \varphi_{\alpha}\right)_{\alpha \in A} \sim\left(V_{\beta}, \psi_{\beta}\right)_{\beta \in B} \Longleftrightarrow\left(U_{\alpha}, \varphi_{\alpha}\right)_{\alpha \in A} \cup\left(V_{\beta}, \psi_{\beta}\right)_{\beta \in B} \in \tilde{K}(X) .
$$

2) We define the space of Kähler forms on $X$ to be the quotient space $\mathcal{K}(X)=\tilde{\mathcal{K}}(X) / \sim$. An element $\omega \in \mathcal{K}(X)$ is called a Kähler form.

3) $(X, \omega)$ is called a Kähler space.

Remark 3.2: One can also give an equivalent definition for a Kähler form on $X$ via differential conditions on the regular locus: a Kähler form is given by $\left(U_{\alpha}, \varphi_{\alpha}\right)_{\alpha}$ with $\varphi_{\alpha}$ strongly plurisubharmonic on $U_{\alpha}$ if any only if

$$
i \partial \bar{\partial}\left(\varphi_{\alpha}-\varphi_{\beta}\right)=0
$$

on $U_{\alpha} \cap U_{\beta} \cap X_{\text {reg. }}$.

Inspired by the characterization given in Remark 3.2, the following definition for LCK spaces was first introduced in [IP], adapting the most wellsuited of the equivalent definitions of LCK manifolds. For technical reasons, we introduce at the same time what we call locally conformally preKähler metrics. 


\section{Definition 3.3:}

1) Denote by

$$
\begin{aligned}
\widetilde{\mathcal{L C K}}(X)=\{ & \left(U_{\alpha}, \varphi_{\alpha}, f_{\alpha}\right)_{\alpha \in A} \mid\left(U_{\alpha}\right)_{\alpha} \text { a covering of } X, \varphi_{\alpha}: U_{\alpha} \longrightarrow \mathbb{R} \\
& \text { strongly plurisubharmonic, } f_{\alpha}: U_{\alpha} \longrightarrow \mathbb{R} \text { smooth and } \\
& \left.e^{f_{\alpha}} i \partial \bar{\partial} \varphi_{\alpha}=e^{f_{\beta}} i \partial \bar{\partial} \varphi_{\beta} \text { on } U_{\alpha} \cap U_{\beta} \cap X_{\text {reg }}, \forall \alpha, \beta \in A\right\} . \\
\widetilde{\mathcal{L C} p \mathcal{K}}(X)=\{ & \left(U_{\alpha}, \varphi_{\alpha}, f_{\alpha}\right)_{\alpha \in A} \mid\left(U_{\alpha}\right)_{\alpha} \text { a covering of } X, \varphi_{\alpha}: U_{\alpha} \longrightarrow \mathbb{R} \\
& \text { plurisubharmonic, } f_{\alpha}: U_{\alpha} \longrightarrow \mathbb{R} \text { smooth and } \\
& \left.e^{f_{\alpha}} i \partial \bar{\partial} \varphi_{\alpha}=e^{f_{\beta}} i \partial \bar{\partial} \varphi_{\beta} \text { on } U_{\alpha} \cap U_{\beta} \cap X_{\mathrm{reg}}, \forall \alpha, \beta \in A\right\} .
\end{aligned}
$$

We define equivalence relations on $\widetilde{\mathcal{L C K}}(X)$ and $\widetilde{\mathcal{L C} p \mathcal{K}}(X)$ by

$$
\begin{array}{r}
\left(U_{\alpha}, \varphi_{\alpha}, f_{\alpha}\right)_{\alpha \in A} \sim\left(V_{\beta}, \psi_{\beta}, h_{\beta}\right)_{\beta \in B} \Longleftrightarrow \\
\left(U_{\alpha}, \varphi_{\alpha}, f_{\alpha}\right)_{\alpha \in A} \cup\left(V_{\beta}, \psi_{\beta}, h_{\beta}\right)_{\beta \in B} \in \widetilde{\mathcal{L C K}}(X) .
\end{array}
$$

and similarly,

$$
\begin{array}{r}
\left(U_{\alpha}, \varphi_{\alpha}, f_{\alpha}\right)_{\alpha \in A} \sim\left(V_{\beta}, \psi_{\beta}, h_{\beta}\right)_{\beta \in B} \Longleftrightarrow \\
\left(U_{\alpha}, \varphi_{\alpha}, f_{\alpha}\right)_{\alpha \in A} \cup\left(V_{\beta}, \psi_{\beta}, h_{\beta}\right)_{\beta \in B} \in \widehat{\mathcal{L C} p \mathcal{K}}(X) .
\end{array}
$$

2) We define the space of locally conformally Kähler (LCK) forms (or metrics) on $X$ to be the quotient space $\mathcal{L C K}(X)=\widetilde{\mathcal{L C K}}(X) / \sim$ and the space of locally conformally preKähler ( $L C p K$ ) forms (or metrics) on $X$ to be the quotient space $\mathcal{L C} p \mathcal{K}(X)=\widetilde{\mathcal{L C} p \mathcal{K}}(X) / \sim$. An element $\omega \in$ $\mathcal{L C}(p) \mathcal{K}(X)$ is called a $\mathrm{LC}(\mathrm{p}) \mathrm{K}$ metric. Obviously, $\mathcal{L C K}(X) \subset \mathcal{L C} p \mathcal{K}(X)$.

3) The covering $\left(U_{\alpha}\right)_{\alpha}$ together with the functions $f_{\alpha}$ give rise to a closed 1 -form $\theta$ on $X$, as described in Definition 2.1. We call $\theta$ the Lee form associated to the $\mathrm{LC}(\mathrm{p}) \mathrm{K}$ metric.

4) $(X, \omega, \theta)$ is called a $L C(p) K$ space.

5) An LCpK metric $\omega$ is called with potential if $\omega=\overline{(X, \varphi, f)}$ for $\varphi: X \longrightarrow$ $\mathbb{R}$ plurisubharmonic and $f: X \longrightarrow \mathbb{R}$ smooth. In this case, we make the notation $\omega=e^{f} \partial \bar{\partial} \varphi$.

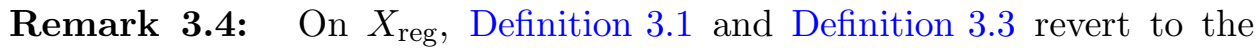
classical definitions for Kähler, locally conformally Kähler and locally conformally preKähler manifolds, respectively.

Remark 3.5: Let $(X, \omega, \theta)$ be an $\mathrm{LC}(\mathrm{p}) \mathrm{K}$ space and $f: X \longrightarrow \mathbb{R}$ a smooth map. Let $\omega$ be given by $\left(U_{\alpha}, \varphi_{\alpha}, f_{\alpha}\right)$. Then $X$ is also $\mathrm{LC}(\mathrm{p}) \mathrm{K}$ with the metric given by $\left(U_{\alpha}, \varphi_{\alpha}, f+f_{\alpha}\right)$, and we denote it by $\left(X, e^{f} \omega, \theta+d f\right)$. Indeed, one can check that its Lee form is $\theta+d f$, with the notations of Definition 2.1.

Remark 3.6: As the notations suggest, if $(X, \omega, \theta)$ is LCK and $\theta=d f$ i.e. is exact, then $\left(X, e^{-f} \omega, \theta-d f=0\right)$ is Kähler. In this case, we call $(X, \omega, \theta)$ globally conformally Kähler (GCK). 
Definition 3.7: Let $\phi: X \longrightarrow Y$ be a holomorphic map between complex spaces and assume $(Y, \omega, \theta)$ is LCpK. Let $\omega=\overline{\left(U_{\alpha}, \varphi_{\alpha}, f_{\alpha}\right)_{\alpha \in A}}$.

Then one sees immediately that $\left(\phi^{-1}\left(U_{\alpha}\right), \phi^{*} \varphi_{\alpha}, \phi^{*} f_{\alpha}\right)_{\alpha \in A}$ gives an LCpK metric on $X$, which we denote by $\phi^{*} \omega$, with Lee form $\phi^{*} \theta$. We call $\phi^{*} \omega$ the pullback of $\omega$ via $\phi$.

As opposed to LCpK forms or closed 1-forms, the pullback of LCK metrics along a holomorphic map is not necessarily LCK. However, there is a particular case where this is true:

Proposition 3.8: Let $\phi: X \longrightarrow Y$ be a a local biholomorphism between complex spaces and assume $(Y, \omega, \theta)$ is $L C K$. Then $\phi^{*} \omega$ is LCK, with Lee form $\phi^{*} \theta$.

Proof. The result is immediate. As $\phi$ is a local biholomorphism, $\phi^{*} \varphi_{\alpha}$ are also strictly plurisubharmonic. In the same way, if $g_{\alpha \beta}$ is holomorphic on $U_{\alpha} \cap U_{\beta}$, then $\phi^{*} g_{\alpha \beta}$ is holomorphic on $\phi^{-1}\left(U_{\alpha}\right) \cap \phi^{-1}\left(U_{\beta}\right)$.

Definition 3.9: Let $(X, \omega, \theta)$ be an $\mathrm{LC}(\mathrm{p}) \mathrm{K}$ space. An automorphism $\gamma \in \operatorname{Aut}(X)$ acts by homothethies of $\omega$ if $\gamma^{*} \omega=e^{c} \omega$ for some $c \in \mathbb{R}$.

We now prove a result giving an alternative characterization for LCK spaces which is a natural extension of the one true in the smooth case and has virtually the same proof, with the help of the notions developed in Section 2. This generalizes a result in [IP]:

Theorem 3.10: Let $X$ be a complex space. Then $X$ admits an LCK metric if and only if its universal covering $\tilde{X}$ admits a Kähler metric such that the deck automorphisms act on $\tilde{X}$ by positive homothethies of the Kähler metric.

Proof. Assume that $(X, \omega, \theta)$ is an LCK space and denote by $\pi: \tilde{X} \longrightarrow X$ the canonical projection. Then $\left(\tilde{X}, \pi^{*} \omega, \pi^{*} \theta\right)$ is an LCK space by Proposition 3.8. Futhermore, by Corollary $2.10, \theta=d f$ for some smooth $f: \tilde{X} \longrightarrow \mathbb{R}$, so Remark 3.6 implies that $\left(\tilde{X}, e^{-f} \pi^{*} \omega\right)$ is Kähler.

Lastly, for any $\gamma$ a deck automorphism of $\tilde{X} \longrightarrow X$, we have $\theta=\gamma^{*} \theta$, so, according to Proposition $2.4, d f=\gamma^{*} d f=d \gamma^{*} f$, so $\gamma^{*} f-f$ is constant. Hence, if

Conversely, assume $(\tilde{X}, \tilde{\omega})$ is Kähler, with the deck group acting by positive homothethies of $\tilde{\omega}$ on it. Then the map

$$
\chi: \operatorname{Deck}(\tilde{X} / X)=\pi_{1}(X) \longrightarrow \mathbb{R}_{>0}, \chi(\gamma)=\frac{\gamma^{*} \tilde{\omega}}{\tilde{\omega}}
$$

is a character of $\pi_{1}(X)$. We can thus construct the line bundle $L=\tilde{X} \times_{\chi} \mathbb{R}$ over $X$ i.e.

$$
L=(\tilde{X} \times \mathbb{R}) /((\tilde{x}, a) \sim(\gamma(\tilde{x}), \chi(\gamma) a)) .
$$

Since there exists a covering of $X$ and a choice of transition maps all of which are positive, $L$ is trivial. Consider a section $v$ of $L$ which is nonzero at every point. Take $\tilde{v}=\pi^{\star}(v)$. Since $L$ is trivial, $\pi^{*} L \longrightarrow \tilde{X}$ is also trivial, 
hence $\tilde{v}$ can be viewed as a function $\tilde{v}: \tilde{X} \rightarrow \mathbb{R}_{>0}$, which can be written $\tilde{v}=e^{-\tilde{f}}$. By the definition of $L$, we have:

$$
\frac{\gamma^{*} \tilde{v}}{\tilde{v}}=\chi(\gamma)=\frac{\gamma^{*} \tilde{\omega}}{\tilde{\omega}}
$$

Consequently, $e^{\tilde{f}} \tilde{\omega}$ is deck invariant, and it descends to a metric $\omega$ on $X$, which, by its definition, is LCK.

\section{Mappings With Discrete FiBers Between LCK SPACES}

In this section, we prove the following

Theorem 4.1: Let $g: X \longrightarrow Y$ be a holomorphic map between complex spaces with discrete fibers and assume $(Y, \omega, \theta)$ is LCK. Then $X$ also carries an LCK structure.

This generalizes a result for Kähler spaces, see [Vaj, Theorem 2]. In fact, our proof consists of a careful intermix of the proof of that result with further topological arguments.

We begin by stating an easy topological fact:

Lemma 4.2: Let $g: X \longrightarrow Y$ be a continuous function with discrete fibers between locally compact topological spaces and choose $x \in U \subset X$ with $U$ open.

Then there exists an open set $W \ni g(x)$ such that the connected component of $g^{-1}(W)$ containing $x$ is contained in $U$.

Definition 4.3: Let $g: X \longrightarrow(Y, \omega, \theta)$ be a holomorphic map between complex spaces with discrete fibers, where $(Y, \omega, \theta)$ is LCK.

We call the coverings $\left(U_{\alpha}\right)_{\alpha \in A}$ of $X$ and $\left(V_{\alpha}\right)_{\alpha \in A}$ of $Y$ well-related if:

1) There exist $\psi_{\alpha}: V_{\alpha} \longrightarrow \mathbb{R}$ strongly plurisubharmonic and $f_{\alpha}: V_{\alpha} \longrightarrow \mathbb{R}$ smooth, such that $\omega=\overline{\left(V_{\alpha}, \varphi_{\alpha}, f_{\alpha}\right)_{\alpha \in A}}$.

2) The covering $\left(U_{\alpha}\right)_{\alpha \in A}$ is locally finite and relatively compact.

3) There exist functions $\varphi_{\alpha}: U_{\alpha} \longrightarrow(0, \infty)$ which are strongly plurisubharmonic.

4) Each $U_{\alpha}$ is a connected component of $g^{-1}\left(V_{\alpha}\right)$.

The existence of such well-related coverings follows from Lemma 4.2.

As we want to use the Kählerianity of the universal covers of $X$ and $Y$ given by Theorem 3.10, we now prove the following technical

Proposition 4.4: Let $g: X \longrightarrow(Y, \omega, \theta)$ be a holomorphic map between complex spaces with discrete fibers, where $(Y, \omega, \theta)$ is $L C K$, and $\left(U_{\alpha}\right)_{\alpha \in A}$ and $\left(V_{\alpha}\right)_{\alpha \in A}$ well-related coverings of $X$ and $Y$ respectively.

Denote by $\pi_{X}: \tilde{X} \longrightarrow X$ and $\pi_{Y}: \tilde{Y} \longrightarrow Y$ the universal coverings of $X$ and $Y$ and consider $\tilde{g}: \tilde{X} \longrightarrow \tilde{Y}$ the lifting of $g$, as in the following commutative diagram: 


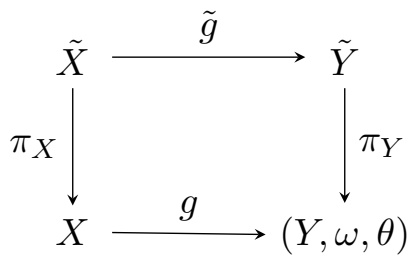

Notice that $\tilde{g}$ also has discrete fibers.

We may also assume that $U_{\alpha}$ are small enough such that $\pi_{X}^{-1}\left(U_{\alpha}\right)$ is a disjoint union of copies of $U_{\alpha}$. For each $\alpha \in A$, choose an isomorphism between the fiber above elements of $U_{\alpha}$ and $\Gamma=\operatorname{Deck}(\tilde{X} / X)$, such that

$$
\pi_{X}^{-1}\left(U_{\alpha}\right)=\coprod_{\gamma \in \Gamma} \tilde{U}_{(\alpha, \gamma)},
$$

with $\pi_{X \mid \tilde{U}_{(\alpha, \gamma)}}: \tilde{U}_{(\alpha, \gamma)} \longrightarrow U_{\alpha}$ a biholomorphism.

For $(\alpha, \gamma) \in A \times \Gamma$, define $\tilde{V}_{(\alpha, \gamma)}=\pi_{Y}^{-1}\left(V_{\alpha}\right)$.

Then the coverings $\left(\tilde{U}_{(\alpha, \gamma)}\right)_{(\alpha, \gamma) \in A \times \Gamma}$ of $\tilde{X}$ and $\left(\tilde{V}_{(\alpha, \gamma)}\right)_{(\alpha, \gamma) \in A \times \Gamma}$ of $\tilde{Y}$ are well-related, where $\left(\tilde{Y}, e^{-f} \pi_{Y}^{*} \omega\right)$ is Kähler, with $d f=\pi_{Y}^{*} \theta$, as proven by Theorem 3.10.

Moreover, given a representation $\rho: \Gamma \longrightarrow \mathbb{R}_{+}$, we may choose $\tilde{\varphi}_{(\alpha, \gamma)}$ : $\tilde{U}_{(\alpha, \gamma)} \longrightarrow(0, \infty)$ strongly plurisubharmonic such that

$$
\eta^{*} \tilde{\varphi}_{(\alpha, \gamma)}=\rho(\eta) \tilde{\varphi}_{\left(\alpha, \eta^{-1} \gamma\right)}, \forall(\alpha, \gamma) \in A \times \Gamma, \forall \eta \in \Gamma .
$$

Proof. We check the conditions imposed by Definition 4.3:

1) As shown in Proposition 3.8 and Theorem 3.10, the choices

$$
\tilde{\psi}_{(\alpha, \gamma)}=\pi_{Y}^{*} \psi_{\alpha}: \tilde{V}_{(\alpha, \gamma)} \longrightarrow \mathbb{R}
$$

are strongly plurisubharmonic and give rise to the Kähler metric $e^{-f} \pi_{Y}^{*} \omega$ on $\tilde{Y}$.

2) The covering $\left(\tilde{U}_{(\alpha, \gamma)}\right)_{(\alpha, \gamma) \in A \times \Gamma}$ is immediately seen to be locally finite and relatively compact.

3) By the definition of well-relatedness, there exist functions $\varphi_{\alpha}: U_{\alpha} \longrightarrow$ $(0, \infty)$ strongly plurisubharmonic. Define

$$
\tilde{\varphi}_{(\alpha, \gamma)}: \tilde{U}_{(\alpha, \gamma)} \longrightarrow(0, \infty), \tilde{\varphi}_{(\alpha, \gamma)}=\rho(\gamma) \pi_{X}^{*} \varphi_{\alpha} .
$$

These functions are obviously strongly plurisubharmonic. Moreover, for an $\eta \in \Gamma, \eta^{*} \tilde{\varphi}_{(\alpha, \gamma)} \in \mathcal{C}^{\infty}\left(\tilde{U}_{\left(\alpha, \eta^{-1} \gamma\right)}\right)$

$$
\begin{aligned}
\eta^{*} \tilde{\varphi}_{(\alpha, \gamma)} & =\rho(\gamma) \eta^{*} \pi_{X}^{*} \varphi_{\alpha}=\rho(\gamma) \pi_{X}^{*} \varphi_{\alpha}=\rho(\eta) \rho\left(\eta^{-1} \gamma\right) \pi_{X}^{*} \varphi_{\alpha} \\
& =\rho(\eta) \tilde{\varphi}_{\left(\alpha, \eta^{-1} \gamma\right)} .
\end{aligned}
$$

4) We need to show that $\tilde{U}_{(\alpha, \gamma)}$ is a connected component of $\tilde{g}^{-1}\left(\tilde{V}_{(\alpha, \gamma)}\right)$, for each $(\alpha, \gamma) \in A \times \Gamma$. Indeed, $\tilde{g}^{-1}\left(\tilde{V}_{(\alpha, \gamma)}\right)=\pi_{X}^{-1}\left(g^{-1}\left(V_{\alpha}\right)\right)$ and, by definition, $U_{\alpha}$ is a connected component of $g^{-1}\left(V_{\alpha}\right)$. 
We can now turn to the

Proof of Theorem 4.1. Denote again by $\pi_{X}: \tilde{X} \longrightarrow X$ and $\pi_{Y}: \tilde{Y} \longrightarrow Y$ the universal coverings of $X$ and $Y$ and consider $\tilde{g}: \tilde{X} \longrightarrow \tilde{Y}$ the lifting of $g$. By Theorem 3.10, $\left(\tilde{Y}, e^{-f} \pi_{Y}^{*} \omega\right)$ is Kähler, where $d f=\pi_{Y}^{*} \theta$.

Pick $\eta \in \Gamma=\operatorname{Deck}(\tilde{X} / X)$. Then

$$
\eta^{*} \tilde{g}^{*}\left(e^{-f} \pi_{Y}^{*} \omega\right)=e^{-\eta^{*} \tilde{g}^{*} f} \eta^{*} \tilde{g}^{*} \pi_{Y}^{*} \omega
$$

(note that this is an equality of LCpK metrics).

But

$$
\eta^{*} \tilde{g}^{*} \pi_{Y}^{*} \omega=\left(\pi_{Y} \tilde{g} \eta\right)^{*} \omega=\left(g \pi_{X} \eta\right)^{*} \omega=\left(g \pi_{X}\right)^{*} \omega=\left(\pi_{Y} \tilde{g}\right)^{*} \omega=\tilde{g}^{*} \pi_{Y}^{*} \omega .
$$

Similarly,

$$
d\left(\eta^{*} \tilde{g}^{*} f\right)=\eta^{*} \tilde{g}^{*} d f=\eta^{*} \tilde{g}^{*} \pi_{Y}^{*} \theta=\tilde{g}^{*} \pi_{Y}^{*} \theta=\tilde{g}^{*} d f=d\left(\tilde{g}^{*} f\right),
$$

so $\eta^{*} \tilde{g}^{*} f=\tilde{g}^{*} f+c_{\eta}$ and thus

$$
e^{-\eta^{*} \tilde{g}^{*} f}=e^{-c_{\eta}} e^{-\tilde{g}^{*} f} .
$$

Using (4.2) and (4.3) in (4.1), we conclude that

$$
\eta^{*} \tilde{g}^{*}\left(e^{-f} \pi_{Y}^{*} \omega\right)=e^{-c_{\eta}} \tilde{g}^{*}\left(e^{-f} \pi_{Y}^{*} \omega\right) .
$$

Note also that $\eta \mapsto e^{-c_{\eta}}$ is a representation of $\Gamma$, which we denote by $\rho$.

For $g: X \longrightarrow(Y, \omega, \theta)$, pick $\left(U_{\alpha}\right)_{\alpha \in A}$ and $\left(V_{\alpha}\right)_{\alpha \in A}$ well-related coverings of $X$ and $Y$ respectively. For $\rho: \Gamma \longrightarrow \mathbb{R}_{+}$chosen as above, we use Proposition 4.4 to construct the well-related coverings $\left(\tilde{U}_{(\alpha, \gamma)}\right)_{(\alpha, \gamma) \in A \times \Gamma}$ of $\tilde{X}$ and $\left(\tilde{V}_{(\alpha, \gamma)}\right)_{(\alpha, \gamma) \in A \times \Gamma}$ of $\tilde{Y}$.

The proof now follows the same broad steps as in [Vaj]. Consider $K_{\alpha} \subset U_{\alpha}$ compact sets with $\bigcup_{\alpha \in A} K_{\alpha}=X$ and choose $\tau_{\alpha} \in \mathcal{C}_{0}^{\infty}\left(V_{\alpha}\right), \tau_{\alpha} \geq 0$ and $\tau_{\alpha}$ equals 1 on a neighborhood of $g\left(K_{\alpha}\right)$. Let

$$
\chi_{(\alpha, \gamma)}= \begin{cases}\left(\tau_{\alpha} \circ g \circ \pi_{X}\right)^{2}=\left(\tau_{\alpha} \circ \pi_{Y} \circ \tilde{g}\right)^{2} & \text { on } U_{(\alpha, \gamma)}, \\ 0 & \text { on } \tilde{X} \backslash U_{(\alpha, \gamma)} .\end{cases}
$$

Note that $\chi_{(\alpha, \gamma)} \varphi_{(\alpha, \gamma)}$ is smooth on $\tilde{X}$ with compact support in $U_{(\alpha, \gamma)}$ and that, for any $\eta \in \Gamma, \eta^{*} \chi_{(\alpha, \gamma)}=\chi_{\left(\alpha, \eta^{-1} \gamma\right)}$.

Then for any $\epsilon=\left(\epsilon_{\alpha}\right)_{\alpha \in A}$ positive numbers, we define the smooth function $\varphi_{\epsilon}: \tilde{X} \longrightarrow(0, \infty)$ by

$$
\varphi_{\epsilon}=\sum_{\substack{\alpha \in A \\ \gamma \in \Gamma}} \epsilon_{\alpha} \chi_{(\alpha, \gamma)} \varphi_{(\alpha, \gamma)}
$$


For an $\eta \in \Gamma$, we have, by Proposition 4.4,

$$
\begin{aligned}
\eta^{*} \varphi_{\epsilon} & =\sum_{\substack{\alpha \in A \\
\gamma \in \Gamma}} \epsilon_{\alpha} \eta^{*}\left(\chi_{(\alpha, \gamma)} \varphi_{(\alpha, \gamma)}\right)=\rho(\eta) \sum_{\substack{\alpha \in A \\
\gamma \in \Gamma}} \epsilon_{\alpha} \chi_{\left(\alpha, \eta^{-1} \gamma\right)} \varphi_{\left(\alpha, \eta^{-1} \gamma\right)} \\
& =\rho(\eta) \varphi_{\epsilon} .
\end{aligned}
$$

Now consider

$$
\phi_{(\alpha, \gamma)}^{\epsilon}: \tilde{U}_{(\alpha, \gamma)} \longrightarrow \mathbb{R}, \phi_{(\alpha, \gamma)}^{\epsilon}=\tilde{g}^{*} \psi_{(\alpha, \gamma)}+\varphi_{\epsilon} .
$$

Since $\phi_{(\alpha, \gamma)}^{\epsilon}-\phi_{(\beta, \sigma)}^{\epsilon}=\tilde{g}^{*}\left(\psi_{(\alpha, \gamma)}-\psi_{(\beta, \sigma)}\right)$, these differences are pluriharmonic.

The fact that one can choose $\epsilon$ and an open covering $\left(U_{(\alpha, \gamma)}^{\prime}\right)_{(\alpha, \gamma) \in A \times \Gamma}$ for which $U_{(\alpha, \gamma)}^{\prime} \subset U_{(\alpha, \gamma)}$ such that $\left(U_{(\alpha, \gamma)}^{\prime}, \phi_{(\alpha, \gamma)}^{\epsilon}\right)_{(\alpha, \gamma) \in A \times \Gamma}$ gives a Kähler form on $\tilde{X}$ now follows from the following Claim, which for clarity we will prove at the end:

Claim: There exists a set $\epsilon^{0}=\left(\epsilon_{\alpha}^{0}\right)_{\alpha \in A}$ of positive constants such that $\phi_{(\alpha, \gamma)}^{\epsilon}$ is strongly plurisubharmonic on the support of $\chi_{(\alpha, \gamma)}$ for all $(\alpha, \gamma) \in$ $A \times \Gamma$ and for all $0<\epsilon<\epsilon^{0}$.

Choose an appropriate $\epsilon$ and denote by $\tilde{\omega}_{X}=\overline{\left(U_{(\alpha, \gamma)}^{\prime}, \phi_{(\alpha, \gamma)}^{\epsilon}\right)_{(\alpha, \gamma) \in A \times \Gamma}}$ the Kähler form on $\tilde{X}$.

For any $\eta \in \Gamma$, we have, by (4.4) and (4.5),

$$
\eta^{*} \tilde{\omega}_{X}=\eta^{*}\left(\tilde{g}^{*} e^{-f} \pi_{Y}^{*} \omega+\partial \bar{\partial} \varphi_{\epsilon}\right)=\rho(\eta)\left(\tilde{g}^{*} e^{-f} \pi_{Y}^{*} \omega+\partial \bar{\partial} \varphi_{\epsilon}\right)=\rho(\eta) \tilde{\omega}_{X},
$$

so $\Gamma=\operatorname{Deck}(\tilde{X} / X)$ acts by positive homothethies of the Kähler metric. By Theorem 3.10, $X$ thus has an LCK form.

We conclude with the proof of the above claim.

Proof of the Claim: We say that a compact subset $L \subset X$ has property $(\star)$ if the following implication holds:

$$
L \cap \operatorname{supp} \chi_{(\alpha, \gamma)} \neq \emptyset \Rightarrow L \subset \operatorname{supp} \chi_{(\alpha, \gamma)} .
$$

For an $L$ with property $(\star)$, denote

$$
\operatorname{index}(L)=\left\{(\alpha, \gamma) \in A \times \Gamma: L \cap \operatorname{supp} \chi_{(\alpha, \gamma)} \neq \emptyset\right\},
$$

which is a nonempty, finite set. We fix an arbitrary point $x_{0} \in X$ and consider $L \subset X$ a compact neighborhood of $x_{0}$ with property $(\star)$. Then, $\tilde{g}(L)$ is also compact and $\tilde{g}(L) \subset \tilde{V}_{(\alpha, \gamma)}$ for all $(\alpha, \gamma) \in \operatorname{index}(L)$.

Next, we want to prove the following assertion:

[†] there exists a constant $\delta=\delta_{L}$ such that $\phi_{(\alpha, \gamma)}^{\epsilon}$ is strongly plurisubharmonic on $L$ for any $0<\epsilon_{\alpha} \leq \delta$ with $\alpha \in \operatorname{index}(L)$, and for any $\gamma \in \Gamma$.

Now, if $L$ is chosen to be sufficiently small, then by using extensions for our functions in some local embeddings, we may consider, without loss of generality, that $\tilde{X}$ and $\tilde{Y}$ are open sets in the Euclidean spaces $\mathbb{C}^{N}$ and $\mathbb{C}^{M}$, respectively. 
Further, for every $(\alpha, \gamma) \in \operatorname{index}(L)$, there exist positive constants $p_{\alpha}, q_{\alpha}$, $b_{\alpha}, c_{\alpha}$ which depend only on $\alpha$, such that

(1) $\mathcal{L}\left(\psi_{\alpha}, \pi_{Y}(\tilde{y})\right) \xi \geq p_{\alpha}\|\xi\|^{2}$,

(2) $\mathcal{L}\left(\varphi_{(\alpha, \gamma)}, \tilde{x}\right) \zeta \geq \rho(\gamma) q_{\alpha}\|\zeta\|^{2}$,

(3) $\left|\varphi_{(\alpha, \gamma)}(\tilde{x}) \mathcal{L}\left(\left(\tau_{\alpha} \circ \pi_{Y}\right), \tilde{y}\right) \xi\right| \leq \rho(\gamma) b_{\alpha}\|\xi\|^{2}$,

(4) $\left|\operatorname{Re} \partial\left(\tau_{\alpha} \circ \pi_{Y}\right)(\tilde{y}) \xi \otimes \bar{\partial} \varphi_{(\alpha, \gamma)}(\tilde{x}) \zeta\right| \leq \rho(\gamma) c_{\alpha}\|\xi\| \cdot\|\zeta\|$,

where $\mathcal{L}$ denotes the Levi form, $\tilde{x} \in L, \tilde{y}=\tilde{g}(\tilde{x}), \zeta \in \mathbb{C}^{N}$, and $\xi=\partial \tilde{g}(\tilde{x}) \zeta$. (1) and (2) hold since $\psi_{\alpha}$ and $\varphi_{(\alpha, \gamma)}$ are strongly plurisubharmonic and $\tilde{g}(L)$, $L$ are compact. (3) and (4) are straightforward.

Computing the Levi form of the function $\phi_{(\alpha, \gamma)}^{\epsilon}$ yields

$$
\mathcal{L}\left(\phi_{(\alpha, \gamma)}^{\epsilon}, \tilde{x}\right) \zeta \geq \rho(\gamma)\left(P\|\xi\|^{2}+Q\|\zeta\|^{2}-B\|\xi\|^{2}-2 C\|\xi\| \cdot\|\zeta\|\right),
$$

where we used the notations

- $P=\min \left\{p_{\alpha}:\right.$ there exists $\gamma$ such that $\left.(\alpha, \gamma) \in \operatorname{index}(L)\right\}$,

- $Q=Q(\tilde{x})=\sum \epsilon_{\alpha} q_{\alpha} \chi_{(\alpha, \gamma)}(\tilde{x})$,

- $B=B(\tilde{x})=2 \sum \epsilon_{\alpha} b_{\alpha} \sqrt{\chi_{(\alpha, \gamma)}(\tilde{x})}$,

- $C=C(\tilde{x})=2 \sum \epsilon_{\alpha} c_{\alpha} \sqrt{\chi_{(\alpha, \gamma)}(\tilde{x})}$.

If we assume for now that

$$
(P-B) Q>C^{2}
$$

holds on $L$, then the right-hand side of (4.6) is positive for all $\zeta \neq 0$. Hence, the existence of the previously mentioned $\delta=\delta_{L}$ is proved.

Now, in order to get (4.7), choose $\delta_{1}>0$ small enough, such that for $0<\epsilon_{\alpha} \leq \delta_{1}$, we have $B \leq P / 2$ on $L$. Then, if $0<\epsilon_{\alpha} \leq \delta_{1}$ for all $\alpha$ with $(\alpha, \gamma) \in \operatorname{index}(L)$, Schwartz's inequality leads to

$$
(P-B) Q \geq \frac{P}{2} \sum \epsilon_{\alpha} q_{\alpha} \chi_{(\alpha, \gamma)} \geq P_{1}\left(\sum \sqrt{\epsilon_{\alpha} q_{\alpha} \chi_{(\alpha, \gamma)}}\right)^{2} .
$$

Since the inequality

$$
\sqrt{\epsilon_{\alpha} q_{\alpha}} \geq \epsilon_{\alpha} \sqrt{\frac{q_{\alpha}}{\delta_{1}}}
$$

is always true, by choosing a convenient (possibly smaller) $\delta_{1}$, we obtain the desired inequality (4.7).

Returning to the main line of proof of our claim, fix $(\alpha, \gamma) \in A \times \Gamma$ and let $L_{1}, L_{2}, \ldots, L_{m}$ be compact subsets of $X$ having the property $(\star)$ such that $(\alpha, \gamma) \in \operatorname{index}\left(L_{j}\right)$ for all $j=1,2, \ldots, m$. Take $\delta_{j}>0$ according to assertion [†] for $L=L_{j}, j=1, \ldots, m$, and set $\delta=\min \left\{\delta_{1}, \ldots, \delta_{m}\right\}$. Then, for any positive numbers $\left\{\epsilon_{\alpha}\right\}$ with $\epsilon_{\alpha} \leq \delta$, for $(\alpha, \gamma) \in \operatorname{index}\left(L_{j}\right), j=1, \ldots, m$ (and no other assumption on $\epsilon_{\beta}$ with $(\beta, \gamma) \notin \cup_{j=1}^{m}$ index $\left(L_{j}\right)$ ), the function $\phi_{(\alpha, \gamma)}^{\epsilon}$ is strongly plurisubharmonic on $\cup_{j=1}^{m} L_{j}$. Assuming we have taken $L_{1}, \ldots, L_{m}$ to cover $\operatorname{supp} \chi_{(\alpha, \gamma)}$, we obtain $\phi_{(\alpha, \gamma)}^{\epsilon}$ strongly plurisubharmonic on $\operatorname{supp} \chi_{(\alpha, \gamma)}$. Moreover, note that $\eta^{*} \phi_{(\alpha, \gamma)}^{\epsilon}=\rho(\eta) \phi_{\left(\alpha, \eta^{-1} \gamma\right)}^{\epsilon}$, hence $\phi_{(\alpha, \eta)}^{\epsilon}$ 
is also strongly plurisubharmonic on $\operatorname{supp} \chi_{(\alpha, \eta)}$ for all $\eta \in \Gamma$, without having to impose further conditions on $\left\{\epsilon_{\alpha}\right\}$. Finally, the local finiteness of $\left\{\operatorname{supp} \chi_{(\alpha, \gamma)}\right\}_{(\alpha, \gamma) \in A \times \Gamma}$ implies that only a finitely many conditions are imposed on every $\epsilon_{\alpha}$, thus concluding the proof of the Claim.

Acknowledgments. We would like to thank Liviu Ornea for his support and suggestions, Victor Vuletescu for helpful discussions about LCK spaces with singularities and Alexandra Otiman for carefully reading the paper and her comments.

\section{REFERENCES}

[G] H. Grauert, Über Modifikationen und exzeptionelle analytische Mengen, Math. Ann. 146 (1962), 331-368. (Cited on page 1.)

[IP] G.-I. Ioniță, O. Preda, LCK metrics on complex spaces with quotient singularities, Manuscripta Math. (2019), DOI:10.1007/s00229-019-01141-w. (Cited on pages 1, 2, 6 , and 8.)

[I] N. Istrati, A characterisation of toric LCK manifolds, to appear in J. Symplectic Geometry, arXiv:1612.03832 (2017). (Cited on page 1.)

[Mo] B. Moishezon, Singular Kählerian spaces, Proceedings of the International Conference on Manifolds and Related Topics in Topology (Tokyo 1973) (1975), 343-351. (Cited on pages 2 and 6.)

[MMOr] A. Moroianu, S. Moroianu, L. Ornea Locally conformally Kähler manifolds with holomorphic Lee field, arXiv:1712.0582 (2017). (Cited on page 1.)

[OV] L. Ornea, M. Verbitsky, A report on locally conformally Kähler manifolds, "Harmonic Maps and Differential Geometry" Contemporary Mathematics 542 (2011), 135-150. (Cited on page 1.)

[Ot] A. Otiman, Morse-Novikov cohomology of locally conformally Kähler surfaces, Math. Z. 289 (2018), Issue 1-2, 605-628. (Cited on page 1.)

[P] P. Pragacz, Contributions to Algebraic Geometry - Impanga Lecture Notes, European Mathematical Society (2012). (Cited on page 2.)

[Vai] I. Vaisman, On locally conformal almost Kähler manifolds, Israel J. Math. 24 No. 3-4 (1976), 338-351. (Cited on page 1.)

[Vaj] V. Vâjâitu, Kählerianity of q-Stein spaces, Arch. Math. 66 (1996), 250-257. (Cited on pages 2,9 , and 11.)

[Var] J. Varouchas, Stabilité de la classe des variétés Kählériennes par certains morphismes propres, Invent. Math. 77, Issue 1, (1984), 117-127. (Cited on page 2.)

Ovidiu Preda

Institute of Mathematics "Simion Stoilow" of the Romanian Academy

21 Calea Grivitei Street, 010702, Bucharest, Romania

E-mail address: ovidiu.preda@imar.ro; ovidiu.preda18@icloud.com

Miron Stanciu

Institute of Mathematics "Simion Stoilow" of the Romanian Academy

21 Calea Grivitei Street, 010702, Bucharest, Romania

E-mail address: miron.stanciu@imar.ro; mirostnc@gmail.com 